\title{
NONLINEAR JOINT ELEMENT FOR THE ANALYSIS OF REINFORCEMENT BARS USING FINITE ELEMENTS
}

\author{
R. Durand ${ }^{1}$, M. M. Farias $^{2}$ \\ ${ }^{1}$ Department of Civil and Environmental Engineering, University of Brasilia (durand@ unb.br) \\ ${ }^{2}$ Department of Geotechnical Engineering, University of Brasilia
}

\begin{abstract}
Reinforcement bars are commonly found in engineering works such as soil nailing, tunnel excavation with the NATM process and in the conventional and pre-stressed reinforced concrete structures. Three approaches are found in the literature for the finite element analysis of such inclusions: the discrete, the smeared and the embedded methods. Each method has its own advantages, however none of them can account for the behavior of the interface between the reinforcement bar and the reinforced medium. The contact simulation is crucial in many cases since the critical failure zone could be the interface itself. This kind of simulation was achieved with the semi-embedded method, proposed by the authors, which combines the advantages of the discrete and embedded methods. In this case reinforcements are composed by real bar elements linked to solid elements by means of joint elements. Each joint element comprises a set of real nodes which are connected to the bar elements and another set of virtual nodes that are linked to particular positions within a trespassed solid element. Joint elements can adopt a nonlinear constitutive model in order to simulate the behavior of an interface failure zone. This paper describes a new and simpler formulation for the semi-embedded method that renders the implementation straightforward. A pull-out test application example is performed showing excellent accuracy.
\end{abstract}

Keywords: Finite elements, Reinforcement, Semi-embedded method.

\section{INTRODUCTION}

Reinforcement bars are commonly used in Geotechnical works such as soil nailing and tunnel excavation with the NATM technique. On the other hand, the finite element method (FEM) is the most popular numerical technique to analyze such structures, due to its versatility to incorporate complex geometries, heterogeneities and advanced constitutive models for geomaterials. Reinforcement bars introduced in the soil mass represent discontinuities which are not easily accounted for using the FEM. Three methods are described in the literature [9] to consider such inclusions: the smeared, the discrete and the embedded methods. The smeared or homogenized method is apparently the simplest. It is employed in cases where 
there is a uniform distribution of reinforcements, so that the elements in this region can be simulated as a new homogeneous material with a different stiffness. However, finding this equivalent stiffness is not a simple matter. The smeared method has been applied in the analysis of reinforced concrete panels, for instance. Nevertheless, this method is not suitable for applications in which the distribution of bars is not uniform, such as in the case of soil nailing with different lengths and inclinations.

The discrete method corresponds to the conventional finite element approach using bar or beam elements. In this case, a new finite element mesh, connecting nodes along the bars to the surrounding soil elements, must be drawn for each reinforcement configuration under analyses during the design stage of a given structure. Furthermore the mesh should be relatively refined along the bar-soil interface. This remeshing can be time consuming and complicated, mainly in three-dimensional analyses as it is the case for bar reinforcements.

The embedded method, on the other hand, allows the reinforcement bars to trespass freely the solid elements. Therefore, a single background mesh can be used to analyze all possible reinforcement configurations under consideration during the design. This is the main advantage of the embedded method. Figure 1 shows a comparison of the required meshes for analyses using the discrete and embedded methods. Details of the embedded method may be found in [5], [8] and [1]. The reinforcement bars are considered by transferring their stiffness to that of surrounding solid elements. In order to achieve this embedment it is generally assumed a perfect adherence between the bars and the involving solid. This is a serious shortcoming, since failure may happen along the bar-soil interface.

In order to account for the relative displacements in the interface between the bars and the surrounding medium, [8], [2] and [6] presented an iterative procedure in which the embedded method is used in a first stage and the results are used to solve a second system considering the bars and joint elements simulated as elasto-plastic springs. The approach works relatively well, but it still has some drawbacks: for instance, it is not possible to apply external boundary conditions directly to the bar nodes.

A new method, called semi-embedded, was introduced by Durand (2008) in order to simulate the interface behavior and to take advantage of the strongest points of the discrete and embedded methods. In this method, the interface is simulated by means of a set of joint elements that link the soil with the reinforcements. These joint elements are represented by non-linear springs located at the nodes of the reinforcement bars and these springs link the reinforcement with the trespassed elements. Since the springs are attached at specific points, this approach will be referred to as "punctual interface modeling".

If the method considers the nodes of the trespassing bars as real nodes that take part in the global system, then it becomes easy to apply direct boundary conditions, such as pull out forces or prescribed displacements. This is an additional advantage of the semi-embedded method in relation to the embedded method in which boundary conditions prescription is rather cumbersome. In addition, by varying the stiffness of the springs in different regions, it is also possible to simulate fully anchored zones and free sliding zones, such as in anchored retaining walls.

The punctual interface approach is still not perfect. The discrete joints do not represent the behavior along the whole interface, but just at the attachment points to which an arbitrary 
proportional area is assigned. Thus, a better approach is desirable in order to take into account all area along the interface. This paper improves the semi-embedded method providing a new formulation where the joint elements are not discrete but continuous.
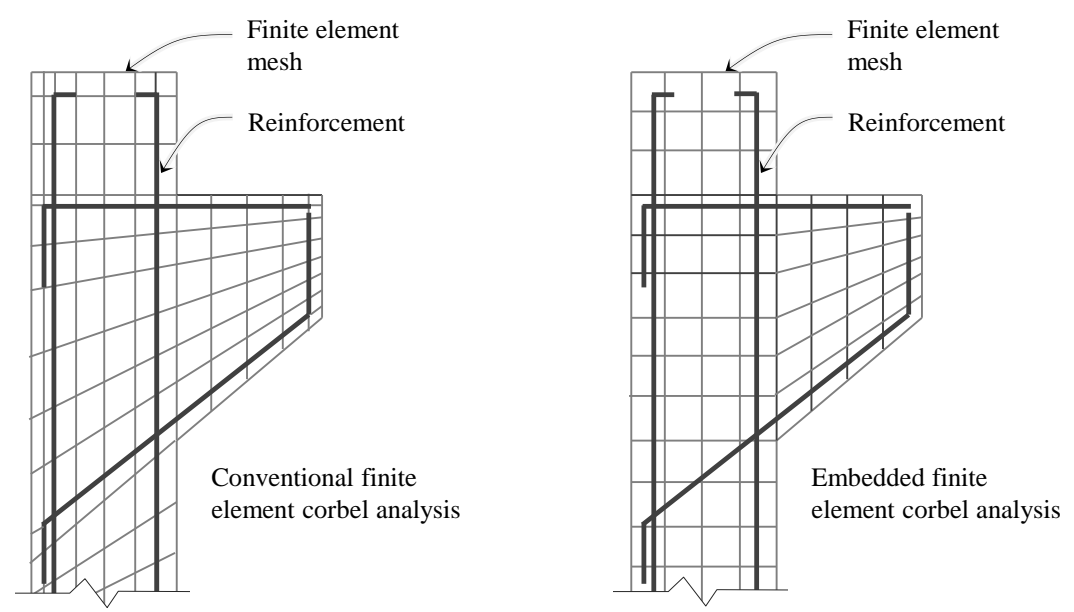

Figure 1. Comparison of finite element meshes for the discrete and embedded element.

\section{THE SEMI-EMBEDDED METHOD}

An entirely new approach called semi-embedded method, which combines features from the discrete and the embedded method, was introduced by [3]. As in the embedded method the reinforcement bars are allowed to trespass the solid elements in any position. However, the bars elements are discrete and have their own independent nodes, which add only a few extra degrees of freedom to the background mesh. Although the bars cross the solid elements, these elements are not split into different regions. This is achieved by superimposing the bars over the solid elements and connecting them by means of springs which act as joint elements. The springs connect the independent extra nodes of the bars to fictitious (or virtual) anchoring nodes along the sides of the solid elements. These virtual nodes do not add any extra degree of freedom to the system and have their displacements computed by interpolation from the nodes of the solid elements, as if they were perfectly adhered to the soil mass.

The reinforcement bars follow the conventional F.E. formulation, but the interface requires special formulation. Two interface formulations will be described in this paper. The first one represents the original formulation introduced by [3] called punctual interface modeling. The second approach represents a new and more comprehensive formulation which will be referred to as "continuous interface modeling".

\subsection{Reinforcement discretization}

Prior to the analysis with the semi-embedded method, all reinforcements are defined by their starting and ending points. During the mesh generation process they should be discretized into bar elements within the bounds of the trespassed elements. In addition, joint elements should be generated in order to link the bar elements with the surrounding soil. 
The nodes of the bar elements are obtained from the intersection between the reinforcement and solid elements as depicted in Figure 2. The iterative algorithm developed by [2] is used in order to define all bar elements. This algorithm finds all intersections between the reinforcement and the surrounding solid elements based on "boundary functions" [2] and a bisection method.

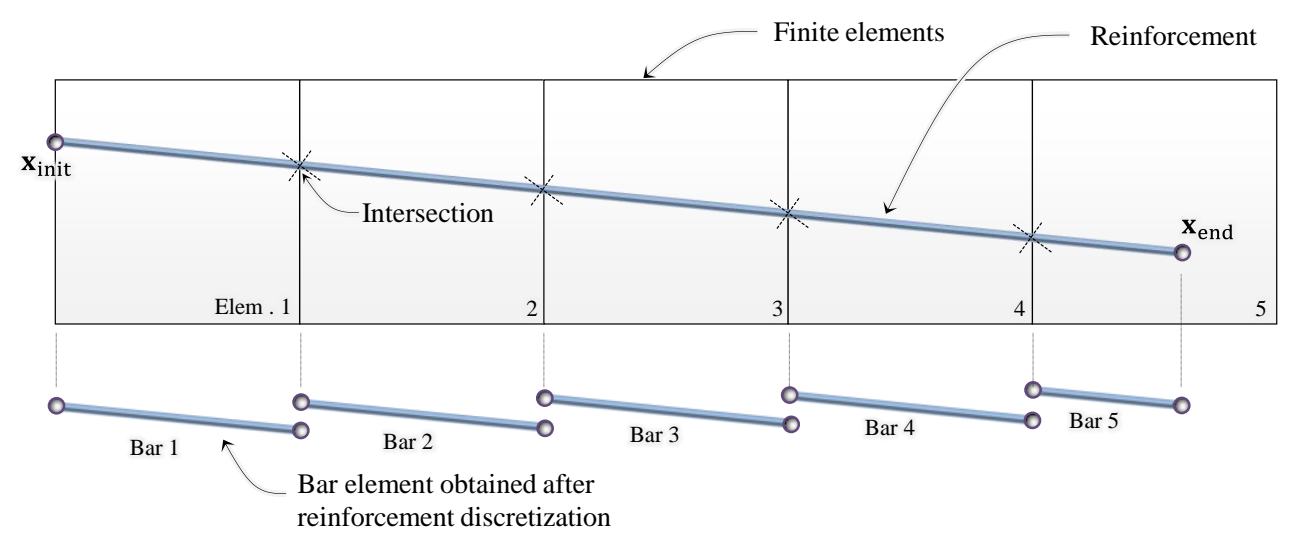

Figure 2. Reinforcement discretization.

The boundary function $\left(f^{b}\right)$ is evaluated at local coordinates $(\eta, \xi, \zeta)$ of a surrounding element and determines if a point is inside $\left(f^{b}>0\right)$, outside $\left(f^{b}<0\right)$ or at the border $\left(f^{b}=0\right)$ of the element. Depending on the shape of a finite element, the boundary function adopts the following expressions:

$$
\begin{gathered}
f_{\text {triangle }}^{b}=\min (\xi, \eta, 1-\xi-\eta) ; \\
f_{\text {quadrilateral }}^{b}=\min (1-\operatorname{abs}(\xi), 1-\operatorname{abs}(\eta)) ; \\
f_{\text {tetrahedron }}^{b}=\min (1-\operatorname{abs}(\xi), 1-\operatorname{abs}(\eta)) ; \\
f_{\text {hexaedron }}^{b}=\min (1-\operatorname{abs}(\xi), 1-\operatorname{abs}(\eta), 1-\operatorname{abs}(\zeta)) .
\end{gathered}
$$

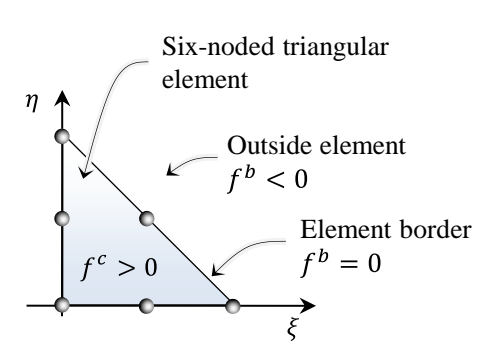

(a)

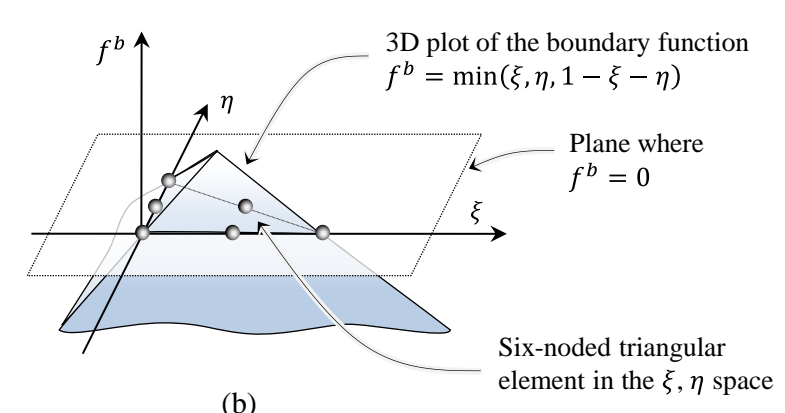

(b)

Figure 3. Boundary function plot for a 2D six-noded triangular element; a) signal variation of the boundary function; b) 3D plot of the corresponding boundary function.

Using the concept of boundary function it is easy to find the relative position of an arbitrary point with respect to a solid element without using extensive geometrical computations. However, for a point given in global $x y z$ coordinates, it is first necessary to find its the 
local coordinates. This can be achieved using the inverse mapping technique ([7],[8] and [2]). Figure 4 shows the graphical interpretation of the inverse mapping.

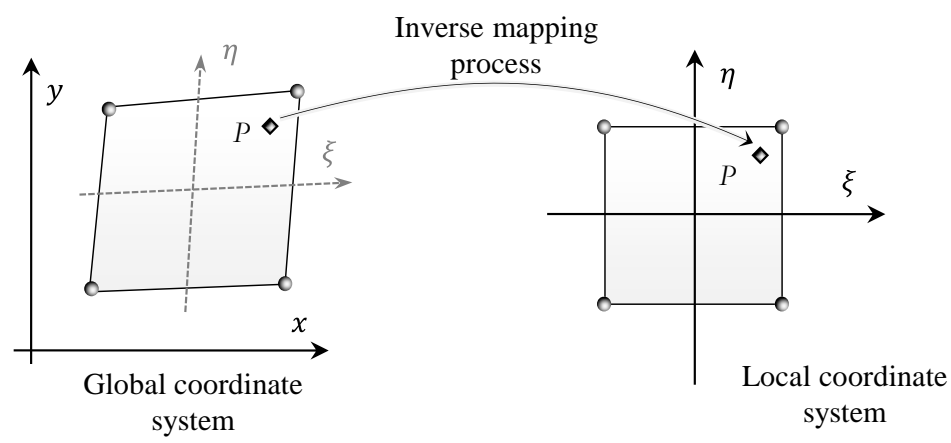

Figure 4. Inverse mapping

An iterative algorithm is used for finding all the intersection points. A virtual point travels back and forth along the reinforcement bar looking for intersection points, where the boundary function evaluated for the surrounding elements is equal to zero. For each intersection point the algorithm uses the bisection method to solve de equation:

$$
f_{\text {current_elem }}^{b}(\eta, \xi, \zeta)=0
$$

Figure 5 shows how the bisection method operates iteratively to find an intersection point between elements 1 and 2. Once the intersection points are found, defining the resulting bar element geometry is straightforward. Bar elements with two or three nodes are commonly used.

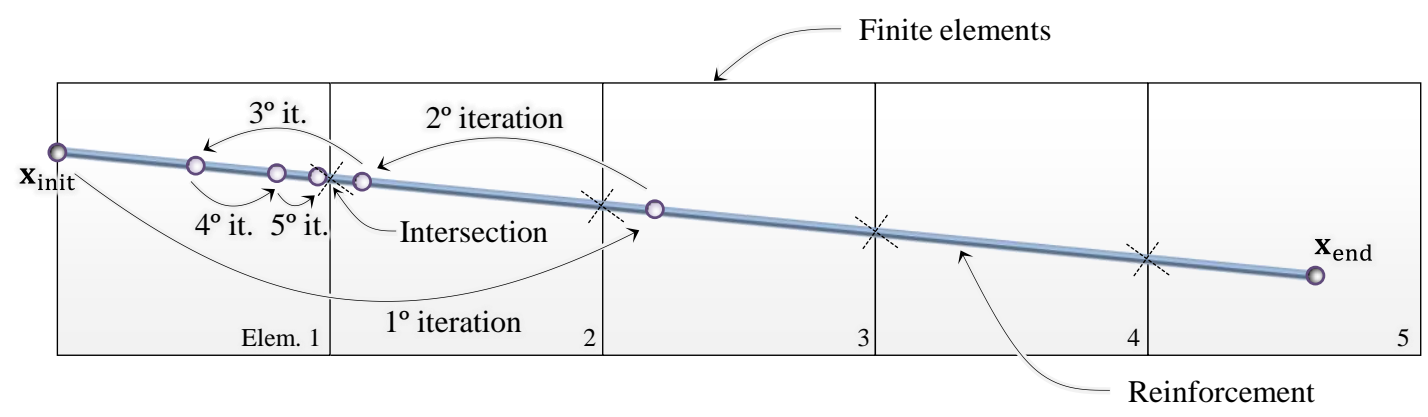

Figure 5. Bisection method for finding intersection points and subsequently the discrete bar elements.

\subsection{Punctual interface modeling}

Bar elements obtained during the reinforcement discretization process should be connected to the system of solid elements in order to compose a single global system. In the punctual interface modeling this is achieved by means of joint elements (springs) representing the interface. The springs have stiffness but no real length, since the real nodes of the bars have the same coordinates as the virtual anchoring nodes in the solid elements. The denomi- 
nation semi-embedded refers to the springs (not the bars) and comes from the fact that only one node of the spring is compatible (embedded) with the displacements of the nodes of the solid element.

Real nodes in the bars have their own equilibrium equations and their displacements are obtained directly when the global system is solved. However, equilibrium of the fictitious nodes on the solid elements is satisfied only in a weak sense and their displacements are computed by interpolation for the displacements of the real nodes of the trespassed solid elements. Since each real node in three-dimensional (3D) problems has three components of displacements, the connections between the bars and the surrounding elements requires three springs at each virtual node, as illustrated in Figure 6. Two of these springs are aligned in the directions perpendicular to the bar axes $\left(y^{\prime}\right.$ and $\left.z^{\prime}\right)$. These springs work like rails or a sheath avoiding significant relative movements between the bars and the surroundings in these directions. The third spring is aligned in the bar direction $x^{\prime}$ and allows the relative displacements of the interface, including possible failure. Depending on the stiffness of this spring, the relative displacements in the interface will mobilize higher or lower stresses levels in the reinforcement bars.

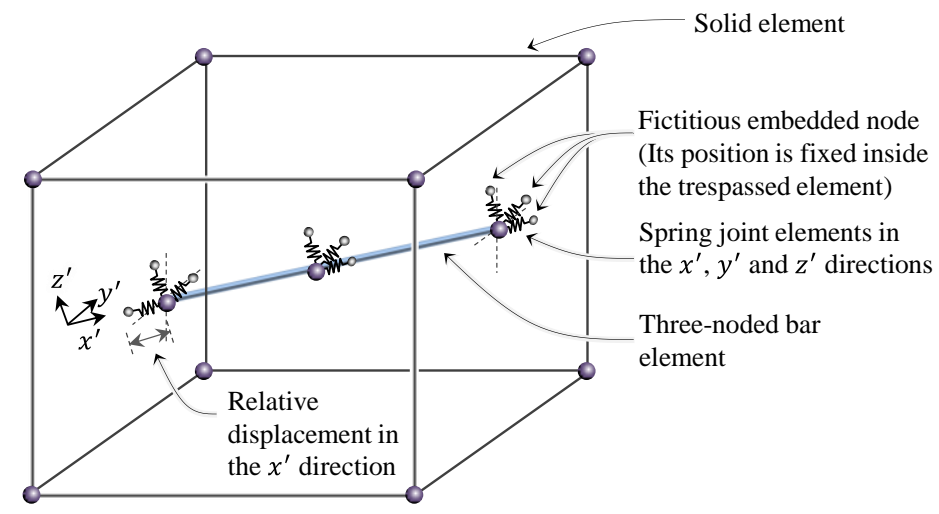

Figure 6. Spring joint elements.

The stiffness of a spring, for instance in the axial bar direction, is determined as a function of the relative displacements between the fictitious anchoring node $\left(P^{*}\right)$ in the solid element and the corresponding real node $(P)$ in the bar element. This is illustrated in Figure 7. The behavior of the interface prior to failure is given by a simple linear relation such as:

$$
\tau=K_{x} \Delta_{x}
$$

in which $\tau$ is the shear stress along the interface, $K_{x}$, is the contact stiffness in the bar direction $\left(x^{\prime}\right)$ and $\Delta_{x}$, represents the relative displacement between the bar and the soil in the axial direction. The contact stiffness is given in units of stress by displacement $[\mathrm{F}][\mathrm{L}]^{-3}$ and may be obtained experimentally from pull-out tests. 


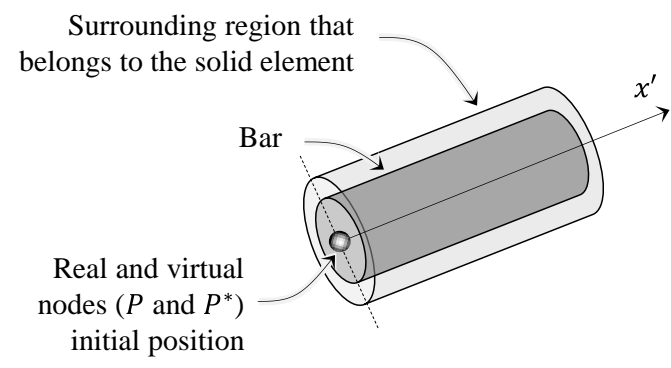

Before deformation

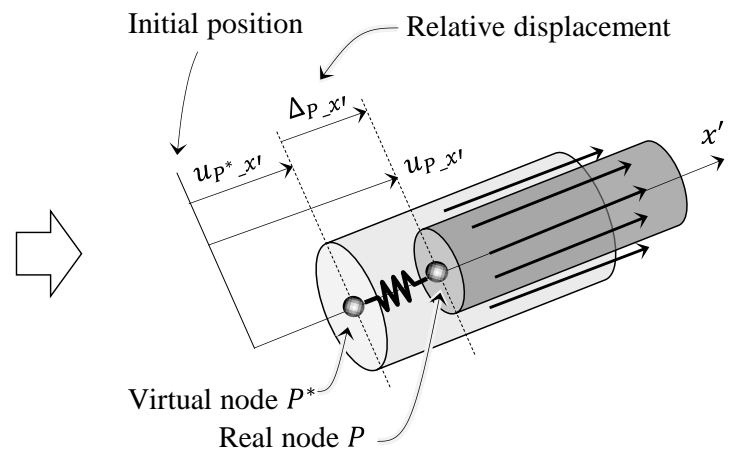

After deformation

Figure 7. Relative displacement between real and virtual nodes along the bar alignment.

The relative movement $\Delta_{x}$ is computed as the difference between the displacement of the real node $(P)$ in the bar and the displacement of the virtual node $\left(P^{*}\right)$, which in turn is computed by interpolation from the displacements of the $m$ real nodes of the trespassed solid element. Therefore, the connecting spring is associated with $(m+1)$ nodes, one node connected to the bar and $m$ connected to the solid element, and the dimension of its stiffness matrix is $3(m+1) \times 3(m+1)$. For the spring in the axial direction $\left(x^{\prime}\right)$, this stiffness is given by the following expression:

$$
\mathbf{K}_{\text {semi_x }}=\mathbf{K}_{x,} A_{\text {lat }} \mathbf{S}_{x^{\prime}}^{T} \mathbf{S}_{x^{\prime}}
$$

in which $A_{\text {lat }}$ is the lateral area of the bar associated to the node (i.e., half of the total bar surface for a two-noded bar element) and $\mathbf{S}_{x}$ is given by:

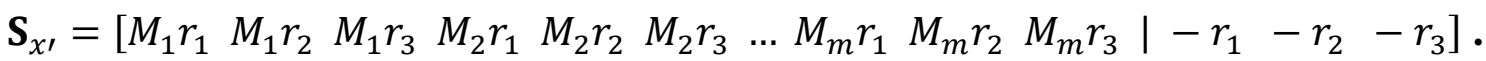

In Eq. 8, $M_{i}$ represent the shape functions of the $m$ nodes of the solid elements and $r_{i}$ are the components of the unit vector in the $x^{\prime}$ direction. The detailed deduction of this equation may be found in [3]. The total stiffness matrix in the contact at an intersection point is given by the addition of the stiffness of the three springs at that point, i.e.:

$$
\mathbf{K}_{\text {joint }}=\mathbf{K}_{\text {joint } x^{\prime}}+\mathbf{K}_{\text {joint } y^{\prime}}+\mathbf{K}_{\text {joint } z^{\prime}} \text {. }
$$

An elastic perfectly plastic behavior may be assumed for the longitudinal spring if one wishes to simulate interface failure in this direction. A suitable constitutive modeling for the interface is explained in section 2.4. If a non-linear stiffness is adopted, the increment of internal forces $\Delta \mathbf{F}_{\text {int }}$ in the interface element may be computed as:

$$
\Delta \mathbf{F}_{\text {joint }}=\mathbf{S}_{x^{\prime}}^{T} \Delta \tau A_{\text {lat }}+\left(\mathbf{K}_{\text {joint } x^{\prime}}+\mathbf{K}_{\text {joint } z^{\prime}}\right) \Delta \mathbf{U}_{\text {joint }}
$$

in which $\Delta \tau$ is the increment in shear stress and $\Delta \mathbf{U}_{\text {joint }}$ is a vector with dimension $3(n+1)$ containing the displacements of the trespassed element and the bar node. 


\subsection{Continuous interface modeling}

The continuous interface modeling represents a variant formulation for the interface element in the semi-embedded method. Whereas the punctual interface modeling considers joint elements constituted by a set of springs placed at the bar nodes, the continuous interface modeling replaces the set of springs by a special single joint element. This special element links a bar element to the corresponding trespassed element without adding extra nodes to the system. Instead of single contact points, the special joint element represents all the contact points in the interface and its stiffness is determined by integration using the Gauss quadrature.

Figure 8 shows the shape of the special joint element. The shaded area represents the possible relative displacements along the contact region. Dark blue circles represent the nodes that connect the solid element and light red circles represent the nodes that connect the bar element. The white nodes are virtual ones whose displacements are calculated by interpolation from the nodes connected to the solid element. At this point, it is clear that the shape and number of nodes of the special joint element is not unique and it depends both on the shape of the trespassed element and the shape of the bar element. Regarding the joint integration, it is performed in one dimension along the contact region over the local coordinate $\xi$ as shown in the figure.

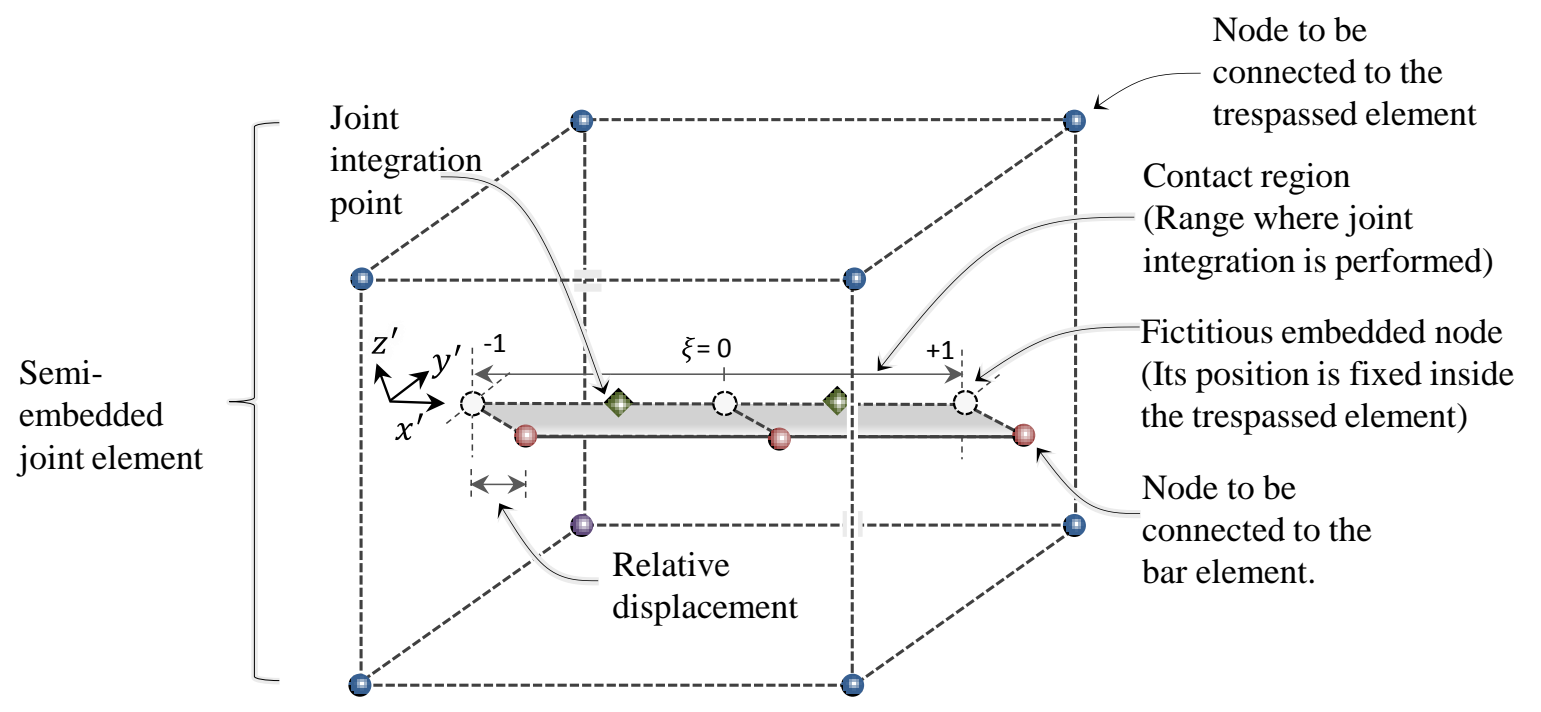

Figure 8. Special joint element.

Relative displacement of a point along the contact region with respect to the local Cartesian coordinate system $x^{\prime} y^{\prime} z^{\prime}$ are expressed in a vector form by $\mathbf{u}_{x^{\prime} y^{\prime} z^{\prime}}^{r}=\left[\begin{array}{lll}u_{x^{\prime}}^{r} & u_{y^{\prime}}^{r} & u_{z^{\prime}}^{r}\end{array}\right]^{T}$. For simplicity, from here the notation $\mathbf{u}^{r \prime}$ will be used instead of $\mathbf{u}_{x \prime y z^{\prime \prime}}^{r}$. It is assumed that the vector $\mathbf{u}^{r \prime}$ can be determined as a function of the nodal displacements of the joint element by means of a transformation matrix $\mathbf{B}$. This relationship can be expressed as:

$$
\mathbf{u}^{r \prime}=\mathbf{B u}
$$


where the joint displacements vector $\mathbf{u}$ comprises all displacements $\mathbf{u}^{t r}$ of the $m$ nodes of the trespassed element plus the displacements $\mathbf{u}^{b}$ of the $n$ nodes of the bar element according to:

$$
\mathbf{u}=\left[\begin{array}{c}
\mathbf{u}^{t r} \\
\mathbf{u}^{b}
\end{array}\right]
$$

Once matrix $\mathbf{B}$ is calculated, the joint element stiffness matrix can be determined in a traditional way as:

$$
\mathbf{K}_{J}=\int \mathbf{B}^{T} \mathbf{D}_{J}^{e p} \mathbf{B} d A
$$

where $\mathbf{D}_{J}^{e p}$ represents the constitutive matrix of the joint material that relates the relative displacements vector $\mathbf{u}^{r^{\prime}}$ with the increments in the contact stress vector $\Delta \boldsymbol{\sigma}=\left[\Delta \tau_{x,} \Delta \sigma_{y,} \Delta \sigma_{z \prime}\right]^{T}$ and $A$ is the area of the cylindrical contact surface. Using the Gauss quadrature and since $d A=P d x^{\prime}$, where $P$ is the perimeter of the contact section, the stiffness matrix can be expressed as the summation along the integration points:

$$
\mathbf{K}_{J}=P \sum_{i=1}^{n \_i p} \mathbf{B}_{i}^{T} \mathbf{D}_{J, i}^{e p} \mathbf{B}_{i}\left|\mathbf{J}_{i}\right| w_{i} .
$$

The matrix $\mathbf{B}$ is fundamental in this formulation and its determination will be outlined now. First, it is considered that the displacement vector $\mathbf{u}^{*}$ at the virtual embedded nodes are obtained as a function of the nodal displacements $\mathbf{u}^{t r}$ of the trespassed element. This relationship can be determined by interpolation using the shape functions $M_{i, j}$ of the solid element. In this case $M_{i, j}$ means the shape function of solid element node $i$ evaluated at the embedded node $j$. Assuming a solid element with $m$ nodes and $n$ embedded nodes in the joint element then:

$$
\left\{\begin{array}{c}
u_{x 1}^{*} \\
u_{y 1}^{*} \\
u_{z 1}^{*} \\
u_{x 2}^{*} \\
u_{y 2}^{*} \\
\vdots \\
u_{x n}^{*} \\
u_{y n}^{*} \\
u_{z n}^{*}
\end{array}\right\}=\left[\begin{array}{ccccccccc}
M_{1,1} & 0 & 0 & M_{2,1} & 0 & \cdots & M_{m, 1} & 0 & 0 \\
0 & M_{1,1} & 0 & 0 & M_{2,1} & \cdots & 0 & M_{m, 1} & 0 \\
0 & 0 & M_{1,1} & 0 & 0 & \cdots & 0 & 0 & M_{m, 1} \\
M_{1,2} & 0 & 0 & M_{2,2} & 0 & \cdots & M_{m, 2} & 0 & 0 \\
0 & M_{1,2} & 0 & 0 & M_{2,2} & \cdots & 0 & M_{m, 2} & 0 \\
\vdots & \vdots & \vdots & \vdots & \vdots & \ddots & \vdots & \vdots & \vdots \\
M_{1, n} & 0 & 0 & M_{2, n} & 0 & \cdots & M_{m, n} & 0 & 0 \\
0 & M_{1, n} & 0 & 0 & M_{2, n} & \cdots & 0 & M_{m, n} & 0 \\
0 & 0 & M_{1, n} & 0 & 0 & \cdots & 0 & 0 & M_{m, n}
\end{array}\right]\left\{\begin{array}{c}
u_{x 1} \\
u_{y 1} \\
u_{z 1} \\
u_{x 2} \\
u_{y 2} \\
\vdots \\
u_{x m} \\
u_{y m} \\
u_{z m}
\end{array}\right\}
$$

The Eq. 15 can be expressed in a condensed matrix form as:

$$
\mathbf{u}^{*}=\mathbf{M u}^{t r}
$$

Assuming a preliminary joint displacement vector of the form $\left[\mathbf{u}^{*} \mathbf{u}^{b}\right]^{T}$, the relative displacement vector in the $x^{\prime} y^{\prime} z^{\prime}$ system evaluated at an arbitrary point along the contact region can be expressed by interpolation as: 


$$
\mathbf{u}^{r \prime}=\mathbf{R}[\mathbf{N}-\mathbf{N}]\left\{\begin{array}{l}
\mathbf{u}^{*} \\
\mathbf{u}^{b}
\end{array}\right\}
$$

where $\mathbf{R}$ is a $3 \times 3$ matrix containing the direction cosines in the directions of $x^{\prime}, y^{\prime}$ and $z^{\prime}$ and $\mathbf{N}$ is a matrix that contains interpolation functions for the contact region which are assumed to be equal to those of the bar element. The matrix $\mathbf{N}$ can be represented in extended form as:

$$
\mathbf{N}=\left[\begin{array}{ccccccccr}
N_{1} & 0 & 0 & N_{2} & 0 & \cdots & N_{n} & 0 & 0 \\
0 & N_{1} & 0 & 0 & N_{2} & \cdots & 0 & N_{n} & 0 \\
0 & 0 & N_{1} & 0 & 0 & \cdots & 0 & 0 & N_{n}
\end{array}\right]
$$

Substituting Eq. 15 into Eq. 17, the following expression is obtained:

$$
\mathbf{u}^{r \prime}=\mathbf{R}\left[\begin{array}{ll}
\mathbf{N} & -\mathbf{N}
\end{array}\right]\left[\begin{array}{c}
\mathbf{M u}^{t r} \\
\mathbf{u}^{b}
\end{array}\right]
$$

which after some rearrangement can be expressed by convenience as:

$$
\mathbf{u}^{r^{\prime}}=\mathbf{R}[\mathbf{N M} \quad-\mathbf{N}]\left[\begin{array}{l}
\mathbf{u}^{t r} \\
\mathbf{u}^{b}
\end{array}\right]
$$

With Eq. 20, a direct relationship between the relative displacements vector and the joint displacement vector $\mathbf{u}=\left[\begin{array}{ll}\mathbf{u}^{t r} & \mathbf{u}^{b}\end{array}\right]^{T}$ is obtained by means of the product $\mathbf{R}[\mathbf{N M}-\mathbf{N}]$ which by comparison with the Eq. 11 represents the desired matrix B. Later, the internal forces in the joint element can be calculated simply as:

$$
\mathbf{F}=\int \mathbf{B}^{T} \boldsymbol{\sigma} d V
$$

or using the Gauss quadrature, as the summation along the integration points:

$$
\mathbf{F}=P \sum_{i}^{n_{-} i p} \mathbf{B}_{i}^{T} \boldsymbol{\sigma}_{i}\left|\mathbf{J}_{i}\right| w_{i}
$$

Two Gauss integration points are recommended for the case of two embedded nodes, and three for the case of three embedded nodes.

\subsection{Interface constitutive modeling}

For a given point in the interface this constitutive modeling aims to find the elastoplastic relationship between the relative displacements vector $\mathbf{u}^{r^{\prime}}=\left[\begin{array}{lll}u_{x^{\prime}}^{r} & u_{y^{\prime}}^{r} & u_{z^{\prime}}^{r}\end{array}\right]^{T}$ (see Figure 9) and the corresponding stress vector in the interface given by $\boldsymbol{\sigma}=\left[\begin{array}{lll}\tau_{x \prime} & \sigma_{y^{\prime}} & \sigma_{z^{\prime}}\end{array}\right]^{T}$. Assuming that the interface failure occurs only along the bar direction $x^{\prime}$, the elasto-plastic modeling will just relate $u_{x^{\prime}}^{r}$ with $\tau_{x^{\prime}}$. These variables will be denoted simply as $u^{r}$ and $\tau$ for the sake of simplicity. For the other variables, assuming an elastic relationship with high stiffness should be enough, since it is considered that relative displacements in the directions $y^{\prime}$ and $z^{\prime}$ are negligible. 


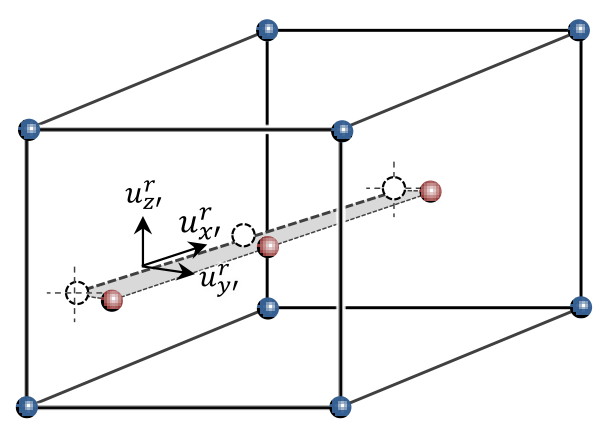

Figure 9. Directions of displacement vector components $u_{r}, u_{y \prime}$ and $u_{z}$.

This derivation will consider a one dimensional elasto-plastic model with some little hardening, similar to a scheme proposed by [10]. In this case, the relative displacement $u^{r}$ plays the role of deformation in the conventional elastic-plastic theory. The interface strength may be given by a criterion similar to that of Mohr-Coulomb (MC), by assuming an adhesion parameter $a$ and a friction angle $\phi$. In this case, the MC failure criterion for the interface can be expressed as:

$$
\tau_{x^{\prime}}=a+\sigma_{c} \tan \phi
$$

where $\tau_{x}$ is the shear stress in the contact region in the bar direction $x^{\prime}$ and $\sigma_{c}$ represents the average confining stress which can be obtained by interpolating the trespassed element stresses as described in section 2.5.

Before failure, a simple elastic behavior can be adopted by means of an interface shear stiffness modulus $K_{J}$ :

$$
\tau_{x^{\prime}}=K_{J} u^{r}
$$

The stiffness modulus $K_{J}$ can be obtained experimentally from pull-out tests according to:

$$
K_{J}=\frac{T}{u_{\max }},
$$

where $T$ and $u_{\max }$ are, respectively, the pull-out force and the final displacement obtained during the test.

Regarding the confining stress $\sigma_{c}$ in Eq. 23, it is worth noting that this value is not a constant and depends on the surrounding stresses level that can change during the analysis. Figure 10 shows two stress-displacement trajectories $A$ and $B$ which are limited by the MC failure criterion. Both trajectories have different confining stress values, so they will reach the failure surface at different points. 


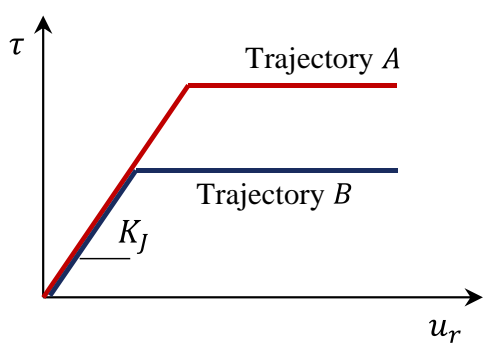

(a)

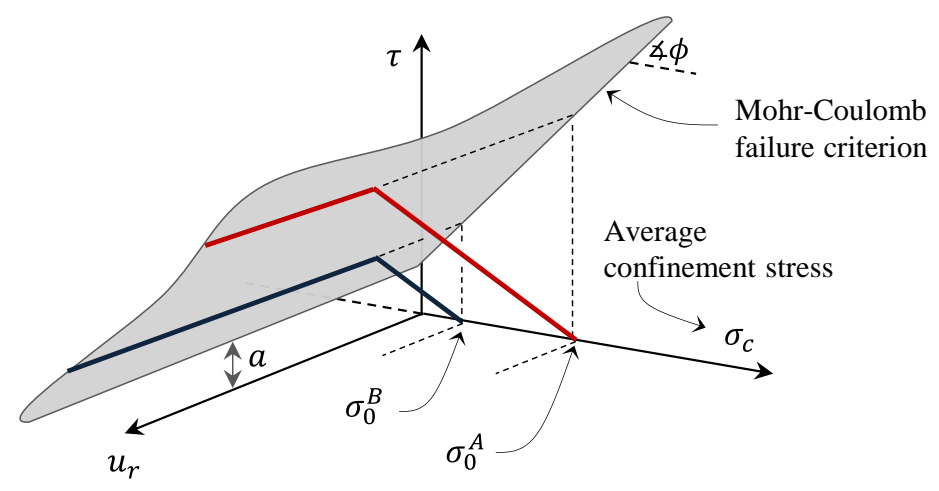

(b)

Figure 10. Stress-displacement trajectories limited by the Mohr-Coulomb failure criterion: a) in the $\tau_{x^{\prime}}-u_{x^{\prime}}^{r}$ space; $\left.\mathrm{b}\right)$ in the $\tau_{x^{\prime}}-u_{x^{\prime}}^{r}-\sigma_{c}$ space.

Following a conventional procedure and taking into consideration the MC failure criterion, the plastic function $F$ can be expressed as:

$$
F=\tau-a-\sigma_{C} \tan \phi+z,
$$

where $z$ represents the hardening parameter. Therefore, the consistency condition is given by:

$$
\dot{F}=\frac{\partial F}{\partial \tau} \dot{\tau}+\frac{\partial F}{\partial z} \dot{z}=0
$$

and assuming an associated flow rule given by $G=F$, the corresponding derivatives are:

$$
\frac{\partial F}{\partial \tau}=1 ; \quad \frac{\partial G}{\partial \tau}=1 ; \quad \frac{\partial F}{\partial z}=1
$$

Considering the relationship $\dot{\tau}=K_{J} \dot{u}_{e}^{r}$, the additive decomposition rule $\dot{u}^{r}=\dot{u}_{e}^{r}+\dot{u}_{p}^{r}$ and the plastic relative displacement expressed as $\dot{u}_{p}^{r}=\Lambda(\partial G / \partial \tau)$, where $\Lambda$ is the plastic multiplier, the shear stress increment can be expressed by:

$$
\dot{\tau}=K_{J}\left(\dot{u}^{r}-\Lambda \frac{\partial G}{\partial \tau}\right)
$$

Taking into account a hardening variable of displacement type which in turn is related with the plastic work $\dot{W}_{p}=\tau \dot{u}_{p}^{r}$, the increment of the internal variable $z$ can be written as:

$$
\dot{z}=H \dot{W}_{p},
$$

where $H$ represents the plastic modulus.

Replacing the Eqs. 27 and 28 into Eq. 29, the plastic multiplier can be expressed as:

$$
\Lambda=\frac{K_{J} \dot{u}^{r}}{K_{J}-H \tau} .
$$

Combining Eqs. 29 and 31 it is possible to obtain an expression for the elastic-plastic joint stiffness $K_{J}^{e p}$ as a function of the elastic joint stiffness $K_{J}$ : 


$$
K_{J}^{e p}=K_{J}-\frac{K_{J}^{2}}{K_{J}-H \tau}
$$

In addition, the increment in the hardening parameter is given by:

$$
\dot{z}=\frac{K_{J} H \tau}{K_{J}-H \tau} \dot{u}^{r}
$$

Since the vector of relative displacements $\mathbf{u}^{r^{\prime}}$ contains components in the three directions, it is convenient to express an elasto-plastic matrix that relates the increment in relative displacements with the joint stress increment:

$$
\left[\begin{array}{c}
\dot{\tau} \\
\dot{\sigma}_{y \prime} \\
\dot{\sigma}_{z \prime}
\end{array}\right]=\left[\begin{array}{ccc}
K_{J}^{e p} & 0 & 0 \\
0 & K_{C} & 0 \\
0 & 0 & K_{c}
\end{array}\right]\left[\begin{array}{c}
\dot{u}^{r} \\
u_{y^{\prime}}^{r} \\
u_{z^{\prime}}^{r}
\end{array}\right],
$$

where $K_{c}$ represent the stiffness in the directions $y^{\prime}$ and $z^{\prime}$ perpendicular to the interface. Finally, the elastic-plastic matrix for the semi-embedded joint element can be written simply as:

$$
\mathbf{D}_{J}^{e p}=\left[\begin{array}{ccc}
K_{J}^{e p} & 0 & 0 \\
0 & K_{C} & 0 \\
0 & 0 & K_{c}
\end{array}\right] .
$$

\subsection{Confining stress determination}

For the case of the reinforcement interface, the conventional MC normal stress is replaced by the confining stress $\sigma_{c}$. For a given point along the interface, e.g. an integration point, the confining stress value can be estimated as a function of the surrounding stresses and therefore its value can change over load increments. Figure 10 shows an idealization of confining stresses over the reinforcement.

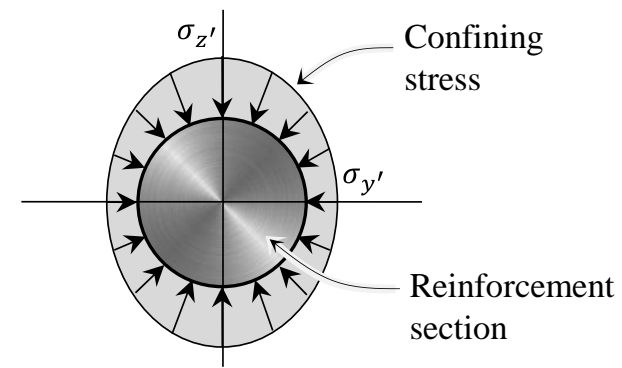

(a)

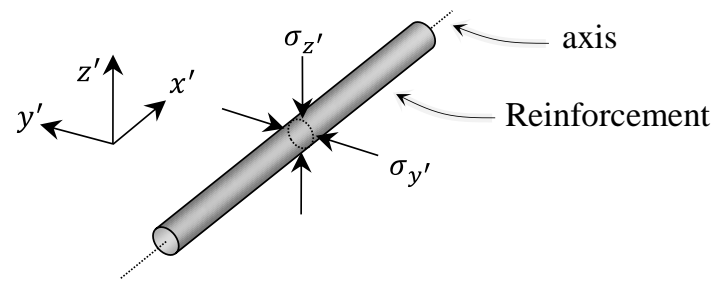

(b)

Figure 11. Bar section showing the confining stresses acting perpendicular to the bar surfaces (a) and principal components of confining stresses at an arbitrary point along the bar (b).

The procedure to obtain the confining stress $\sigma_{c}$ as a function of the trespassed element stresses is explained as follows. First, for a given point, with local coordinate $\xi$ along the interface, its corresponding global coordinates $x y z$ should be found. This task is trivial and 
easily done by interpolation of the embedded nodal coordinates. After the global coordinates of the point are found, they should be converted to local coordinates $\eta \xi \zeta$ related to the trespassed element. For these coordinates, the stress vector $\boldsymbol{\sigma}_{x \prime y^{\prime} z^{\prime}}$ could be found by a process of extrapolation and interpolation from the stresses in the trespassed element $\boldsymbol{S}_{i p}$ according to:

$$
\boldsymbol{\sigma}_{x y z}=\left(\mathbf{N}(\xi, \eta, \zeta)^{T} \mathbf{E} \boldsymbol{\sigma}_{i p}\right)^{T}
$$

where $\boldsymbol{\sigma}_{i p}$ is a matrix containing the stresses from all integration points of the trespassed element:

$$
\boldsymbol{\sigma}_{i p}=\left[\begin{array}{cccccc}
\sigma_{x}^{i p 1} & \sigma_{y}^{i p 1} & \sigma_{z}^{i p 1} & \tau_{x y}^{i p 1} & \tau_{y z}^{i p 1} & \tau_{x z}^{i p 1} \\
\sigma_{x}^{i p 2} & \sigma_{y}^{i p 2} & \sigma_{z}^{i p 2} & \tau_{x y}^{i p 2} & \tau_{y z}^{i p 2} & \tau_{x z}^{i p 2} \\
\sigma_{x}^{i p 3} & \sigma_{y}^{i p 3} & \sigma_{z}^{i p 3} & \tau_{x y}^{i p 3} & \tau_{y z}^{i p 3} & \tau_{x z}^{i p 3} \\
\vdots & \vdots & \vdots & \vdots & \vdots & \vdots \\
\sigma_{x}^{i p m} & \sigma_{y}^{i p m} & \sigma_{z}^{i p m} & \tau_{x y}^{i p m} & \tau_{y z}^{i p m} & \tau_{x z}^{i p m}
\end{array}\right]
$$

$\mathbf{E}$ is an extrapolation matrix that allows the determination of nodal values from the values given at integration points (see [4]). $\mathbf{N}$ is a vector containing the trespassed element shape functions evaluated at local coordinates $\eta, \xi$, and $\zeta$.

The stresses vector given by Eq. 36 can be transformed into the $x^{\prime} y^{\prime} z^{\prime}$ system by a conventional change in coordinates systems by means of a matrix $\mathbf{T}$ according to:

$$
\left\{\begin{array}{c}
\sigma_{x^{\prime}} \\
\sigma_{y^{\prime}} \\
\sigma_{z^{\prime}} \\
\tau_{x^{\prime} y^{\prime}} \\
\tau_{y^{\prime} z^{\prime}} \\
\tau_{x^{\prime} z^{\prime}}
\end{array}\right\}=\left[\begin{array}{cccccc}
l_{1}^{2} & m_{1}^{2} & n_{1}^{2} & 2 l_{1} m_{1} & 2 m_{1} n_{1} & 2 n_{1} l_{1} \\
l_{2}^{2} & m_{2}^{2} & n_{2}^{2} & 2 l_{2} m_{2} & 2 m_{2} n_{2} & 2 n_{2} l_{2} \\
l_{3}^{2} & m_{3}^{2} & n_{3}^{2} & 2 l_{3} m_{3} & 2 m_{3} n_{3} & 2 n_{3} l_{3} \\
l_{1} l_{2} & m_{1} m_{2} & n_{1} n_{2} & l_{1} m_{2}+l_{2} m_{1} & m_{1} n_{2}+m_{2} n_{1} & l_{1} n_{2}+l_{2} n_{1} \\
l_{2} l_{3} & m_{2} m_{3} & n_{2} n_{3} & l_{2} m_{3}+l_{3} m_{2} & m_{2} n_{3}+m_{3} n_{2} & l_{2} n_{3}+l_{3} n_{2} \\
l_{3} l_{1} & m_{3} m_{1} & n_{3} n_{1} & l_{3} m_{1}+l_{1} m_{3} & m_{3} n_{1}+m_{1} n_{3} & l_{3} n_{1}+l_{1} n_{3}
\end{array}\right]\left\{\begin{array}{c}
\sigma_{x} \\
\sigma_{y} \\
\sigma_{z} \\
\tau_{x y} \\
\tau_{y z} \\
\tau_{x z}
\end{array}\right\},
$$

where $l, m$ and $n$ are scalars representing the directional cosines between the $x y z$ and $x^{\prime} y^{\prime} z^{\prime}$ coordinate systems. Applying this coordinates transformation in Eq. 36 the following expression is obtained:

$$
\boldsymbol{\sigma}_{x \prime y^{\prime} z^{\prime}}=\mathbf{T}\left(\mathbf{N}(\xi, \eta, \zeta)^{T} \mathbf{E} \boldsymbol{\sigma}_{i p}\right)^{T}
$$

\section{APPLICATION}

A pull-out test is performed in order to evaluate if the interface resistance is in accordance with the assumed interface strength parameters. Figure 12 shows the test configuration. Eight-noded brick elements with $2 \times 2 \times 2$ integration were used and a single inclined reinforcement bar with $4 \mathrm{~m}$ in length was introduced across the mesh. Three-noded bar elements were generated automatically by a mesh generator program, as well as the semi-embedded joint elements. 


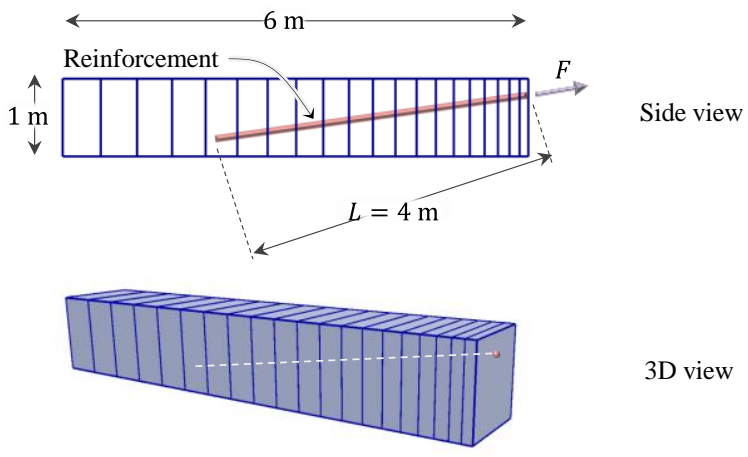

Figure 12. Finite element mesh and reinforcement in a pull-out test.

The bar reinforcement has an Young Modulus $E=210 \mathrm{GPa}$ and a cross section area $A=0.005 \mathrm{~m}^{2}$. The longitudinal interface stiffness is $K_{J}=100 \mathrm{MPa} / \mathrm{m}$ and the interface strength parameters are $a=10 \mathrm{kPa}$ and $\phi=30^{\circ}$. The stiffness perpendicular to the reinforcement is $K_{c}=10 \times 10^{4} \mathrm{MPa} / \mathrm{m}$. Due to the presence of grouting, the interface perimeter is assumed to be equal to $=0.4 \mathrm{~m}$.

An initial isotropic confining stress equal to $100 \mathrm{kPa}$ is imposed to all elements. This implies an estimated shear strength, $\tau_{\max }=a+\sigma_{c} \tan (\phi)$, of $67.73 \mathrm{kPa}$ and an estimated pull out force, $F_{\max }=P L \tau_{\max }$, of $108.95 \mathrm{kN}$. The displacements of all solid nodes are clamped in order to force the relative displacements along the bar-soil interface and the pull out force is applied in increments of $1 \%$ of total force until reaching complete failure.

Figure 13 shows the ratio $\left(\tau / \tau_{\text {máx }}\right)$, i.e., the mobilized shear stress normalized by the predicted shear strength, for different levels of relative external pull out force $\left(F / F_{\text {máx }}\right)$ shown in the legend of the figure. It can be observed that the shear strength of the interface is gradually mobilized along the bar length. Failure initializes close to the pull out node and spreads gradually towards the other extremity of the reinforcement. Complete interface failure was achieved when the external force achieved the forecasted maximum force. This result represents an excellent accuracy obtained by the continuous interface formulation.

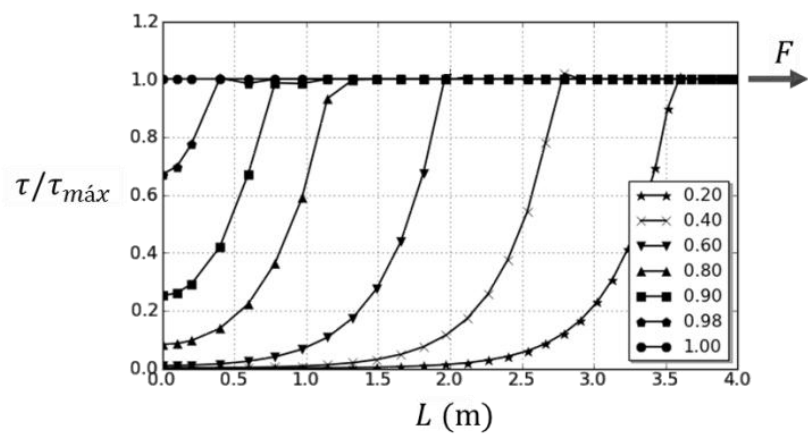

Figure 13. Mobilized shear ratio with external load application.

\section{CONLCUSIONS}

This paper introduces a new formulation for semi-embedded technique for the simulation of reinforcements using the finite element method presented by [3] and described in sec- 
tion 2.2. The main advantage of this new formulation is that the interface between a bar and the reinforced medium can be simulated as a continuum using a single special interface element instead of a set of springs.

In addition to the neater formulation, the proposed changes make implementation and further development much simpler and straightforward.

The method can be applied in 2D or 3D and it is independent on the element type or constitutive behavior of the solid background elements. In this paper, it was tested for a simple pull-out test with excellent accuracy.

\section{REFERENCES}

[1] Andrade, H.A.C., "Numerical procedures for the analisys of drainage elements is soils". MSc Dissertation, Pontifical Catholic University of Rio de Janeiro, Rio de Janeiro, Brazil, 125 p, 2003.

[2] Durand, R., "Embedded stiffness method in the tree-dimensional analysis of reinforcements via finite elements”. MSc Dissertation, University of Brasilia, Brasilia, Brazil, 95 p., 2003.

[3] Durand, R., "Three-dimensional analysis of geotechnical structures subjected to reinforcement and drainage". PhD Thesis, University of Brasilia, Brasilia, Brazil, 153 p, 2008.

[4] Durand, R., Farias, M.M., "A generalized method for the extrapolation of internal values to the nodal points of finite elements". In: 2nd International Workshop in Computational Geotechnics, IWS-Fortaleza, 1, 21-28, 2004.

[5] Elwi, A.E., Hrudey, T.M., "Finite element model for curved embedded reinforcements". Engineering Mechanics, ASCE, 115(4), 740-754, 1989.

[6] Farias, M. M., Durand, R., "Embedded stiffness approach to compute the optimum configuration of reinforcements". In: NUMOG IX, International Symposium on Numerical Models in Geomechanics, 1, 549-555, 2004.

[7] Farias, M.M., Naylor, D.J., "Safety analysis using finite elements". Computers and Geotechnics, Elsevier Science, 22(2), 165-181, 1998.

[8] Hartl, H., "Development of a continuum-mechanics-based tool for 3D finite element analysis of reinforced concrete structures and application to problems of soil-structure interaction", PhD Thesis, Graz University of Technology, Graz, Austria, 250 p, 2002.

[9] Kwak, H.G., Filippou, F.C., "Finite element analysis of reinforced concrete structures under monotonic loads". Report No. UCB/SEMM-90/14, Department of Civil Engineering, University of California, Berkeley, USA, 120 p, 1990.

[10] Nakai, T., "Finite element computations for active and passive earth pressure problems of retaining wall”. Soils and Foundations, 25(3), 98-11, 1985. 